\title{
On the effect of curved streamlines on the accuracy of PIV vector fields
}

\author{
Sven Scharnowski · Christian J. Kähler
}

Received: 21 May 2012/Revised: 16 October 2012/ Accepted: 10 December 2012/Published online: 27 December 2012

(C) The Author(s) 2012. This article is published with open access at Springerlink.com

\begin{abstract}
This article derives a method to estimate and correct the bias error of the shift vector's absolute length in the presence of curved streamlines. The main idea is to identify the most likely streamline with constant curvature from the second-order shift vector and its gradient. The work establishes a theoretical framework including the systematic errors of the first-order and second-order shift vector's absolute value and angle. Synthetic images of a stationary vortex are used to validate the proposed method. The curvature-correction is also applied to a synthetic flow field with non-constant curvature to demonstrate its potential for more realistic flow fields. The results reveal that second-order accurate vector fields suffer from a biased shift vector length depending on the streamline's curvature and on the shift vector length. The bias error is negligible for vector fields with a shift vector length below the streamline curvature radius. For large shift vectors or strong curvatures, the bias error can be significantly reduced with the developed method. The approach is very general and can be applied to any vector field obtained from window-correlation particle image velocimetry (PIV), single-pixel ensemble-correlation PIV, particle tracking velocimetry or optical flow methods. It also works for all 3D extensions of the techniques, such as 3D-PTV or tomographic PIV.
\end{abstract}

S. Scharnowski $(\square) \cdot$ C. J. Kähler

Institute of Fluid Mechanics and Aerodynamics,

Universität der Bundeswehr München,

85577 Neubiberg, Germany

e-mail: sven.scharnowski@unibw.de

\section{Introduction}

Particle image velocimetry (PIV) is a non-intrusive measurement technique that estimates the first-order velocity field in a plane, or even in a volume, by measuring the displacement of particles in a certain time interval $\Delta t$. Due to the nature of the recording principle, each measured velocity vector represents a volume-averaged mean motion of the discretized and quantized tracer particle's diffraction images, rather than the actual velocity of the flow (Adrian and Westerweel 2010; Raffel et al. 2007). To maximize the bandwidth of PIV measurements, the dynamic spatial range (DSR) and the dynamic velocity range (DVR) must be maximized (Adrian 1997).

Single-pixel ensemble-correlation allows for the reduction in the averaging volume in two dimensions, compared to standard window-correlation (Westerweel et al. 2004; Kähler et al. 2006; Scharnowski et al. 2012). Thus, singlepixel ensemble-correlation yields a very large DSR, in particular for low-magnified images (Kähler et al. 2012).

Eckstein and Vlachos (2009) as well as Sciacchitano et al. (2012) presented methods to improve the accuracy of PIV evaluation, which results in increased DVR values. Another possibility to achieve a large DVR, as well as to get accurate estimations of the velocity and quantities derived from it, especially for low magnifications, is to maximize the particle image shift by selecting a sufficiently large time delay between subsequent illuminations. On the other hand, a large time separation may cause a bias error in areas with curved streamlines, as illustrated in Fig. 1. Wereley and Meinhart (2001) analyzed a method for the estimation of the shift vectors with second-order accuracy, and Scholz and Kähler (2006) applied this method to singlepixel vector fields. To achieve an estimation with higher-order accuracy, more than two time steps are generally required 


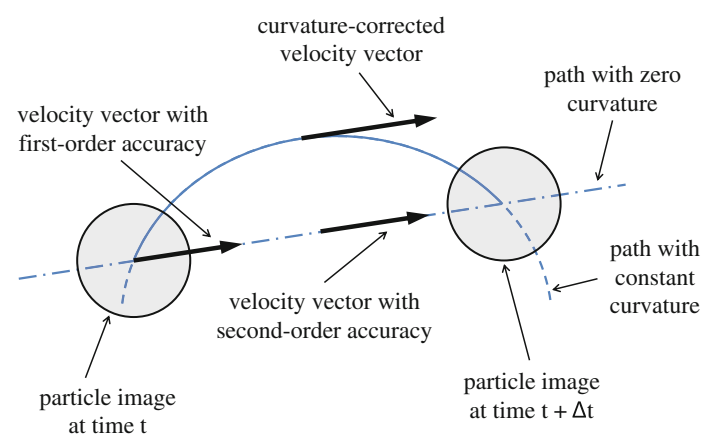

Fig. 1 Particle path estimated from the position of the particle image at time $t$ and $t+\Delta t$

(Lele 1991). A multi-pulse system, as outlined in Kähler and Kompenhans (2000), can be combined with a multiframe evaluation method. Hain and Kähler (2007) as well as Persoons and O'Donovan (2011) describe such a method, developed for PIV, and Cierpka et al. (2012) recently reported an analogous idea, applicable to PTV. Using either combination would allow for higher-order accuracy velocity measurements.

Since multi-pulse techniques require more expensive equipment, the idea of this paper is to achieve higher-order accuracy from conventional double-pulse recordings by estimating the curvature of the particle path from the second-order shift vector field. The reconstruction of the particle path from the local shift vectors and their gradients is mainly based on the assumption that neighboring streamlines do not cross. The article is divided into three main sections. Section 2 discusses the curvature-correction from an analytical point of view. In Sects. 3 and 4, the developed method is applied to synthetic PIV data of a flow fields with constant and non-constant curvature, respectively, in order to verify the theoretical predictions. Finally, the approach is tested on a realistic flow field from the third PIV challenge (Sect. 5)

\section{Mathematical description}

\subsection{First-order bias error}

The evaluation of double-pulse double-frame PIV images approximates the path of motion by a straight line, and the first-order shift vector simply connects the location of particle images at time $t$ and at time $t+\Delta t$. The straight line is the shortest possible path; thus, for complex flows, the actual path is usually longer. Hence, the absolute value of the estimated shift vector is generally underestimated. Assuming an actual path with constant curvature, as shown in Fig. 2, this bias error can be computed from the difference of the arc length and the secant

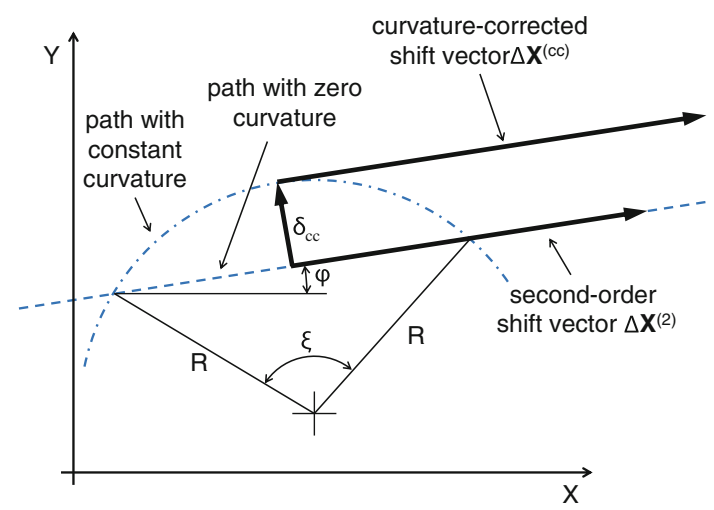

Fig. 2 Second-order shift vector and curvature-corrected shift vector

$$
\begin{aligned}
\delta_{\mathrm{abs}} \Delta \mathbf{X}^{(1)} & =\xi \cdot R-\left|\Delta X^{(1)}\right| \\
& =R\left(\xi-2 \cdot \sin \frac{\xi}{2}\right)
\end{aligned}
$$

where $R$ is the radius of the curvature, $\left|\Delta X^{(1)}\right|$ is the absolute value of the first-order shift vector and $\xi$ the arc's angle (see Fig. 2). Furthermore, the angle of the estimated first-order shift vector is biased if the actual path is curved

$\delta_{\text {angle }} \Delta \mathbf{X}^{(1)}=\frac{\pi}{2}-\arccos \left(\frac{\left|\Delta X^{(1)}\right|}{2 \cdot R}\right)$

In Eq. 2.2, $\pi / 2$ is the angle between the tangent of the arc and the radius, whereas the arccos term is the angle between the first-/second-order shift vector and the radius. For $|\xi|<\pi$, Eq. 2.2 can be reduced to

$\delta_{\text {angle }} \Delta \mathbf{X}^{(1)}=\frac{\xi}{2}$

Both the bias of the absolute value and the bias of the angle depend on the radius of the curved path and on the arc's angle. However, these quantities are unknown and cannot be measured directly.

\subsection{Second-order bias error}

For second-order accurate PIV evaluation, the first-order shift vector is moved to the middle position of the start point and the end point of the vector. This fully compensates for the angular error from Eq. 2.2 in the case of constant curvature, since the vector is aligned tangentially to the actual path. However, the vector's length is still biased. Furthermore, the vector is pushed to a different streamline where the actual velocity is generally different. The bias error of the absolute velocity depends on the curvature, on the arc's angle and on the change of the second-order shift vector's absolute value perpendicular to the streamline 


$$
\begin{aligned}
\delta_{\mathrm{abs}} \Delta \mathbf{X}^{(2)}= & \xi \cdot R-\left|\Delta X^{(2)}\right|+\left|\boldsymbol{\delta}_{\mathrm{cc}}\right| \cdot\left|\frac{\partial\left|\Delta \mathbf{X}^{(2)}\right|}{\partial \mathbf{n}}\right| \\
= & R \cdot\left(\xi-2 \cdot \sin \frac{\xi}{2}\right) \\
& +R \cdot\left(1-\cos \frac{\xi}{2}\right) \cdot\left|\frac{\partial\left|\Delta \mathbf{X}^{(2)}\right|}{\partial \mathbf{n}}\right|
\end{aligned}
$$

where $\mathbf{n}$ is the unit vector perpendicular to the streamline. As before, the bias depends on the unknown parameters $\xi$ and $R$.

\subsection{Curvature-correction}

In order to compensate for first- and second-order bias errors, the parameters $\xi$ and $R$ must be extracted from the shift vector field. Neighboring shift vectors that connect two points on the same streamline can be used to estimate the flow path's curvature, as illustrated in Fig. 3. For the sake of clarity, the second-order shift vector in Fig. 3 is moved such that its center falls on the original starting point. The same holds for the neighboring vector and for the curvature-corrected one.

For the curvature-correction, the main idea is to find a neighboring vector in the vicinity $(r \rightarrow 0$, see Fig. 3 ) of the second-order shift vector such that both vectors connect two points on the same circle. Under this condition, the following relation holds between the angles of the vectors $\boldsymbol{\delta}_{\mathbf{1}}$ and $\boldsymbol{\delta}_{\mathbf{2}}$ and the second-order shift vector

$\beta_{1}+\phi-\phi_{n}=\beta_{2}$

Where $\phi$ and $\phi_{n}$ are the angle of the second-order shift vector and the neighboring vector. The starting point of the neighboring vector must be close to the starting point of the shift vector in order to align the vector $\boldsymbol{\delta}_{\mathbf{1}}$ tangentially to the circle. Due to the finite vector spacing of PIV data

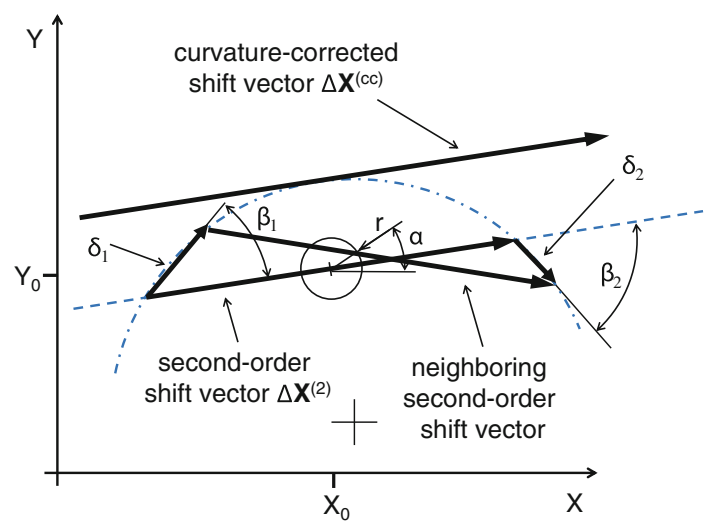

Fig. 3 Curvature-correction by using neighboring, which are connected by the same streamline points, a local (first-order) series expansion for the neighboring vector

$\Delta \mathbf{X}^{(2)}\left(\left(X_{0}, Y_{0}\right)+\boldsymbol{\delta}_{1}\right)=\Delta \mathbf{X}^{(2)}\left(X_{0}, Y_{0}\right)+\frac{\partial \Delta \mathbf{X}^{(2)}}{\partial \boldsymbol{\delta}_{1}} \cdot \boldsymbol{\delta}_{\mathbf{1}}+\cdots$

is required in order to interpolate between the discrete data points. The second-order shift vector $\Delta \mathbf{X}^{(2)}\left(X_{0}, Y_{0}\right)$ and a neighboring shift vector that fulfills the condition in Eq. 2.5 differ by the vector $\boldsymbol{\delta}_{\mathbf{2}}-\boldsymbol{\delta}_{\mathbf{1}}$ :

$\Delta \mathbf{X}^{(2)}\left(X_{0}, Y_{0}\right)+\boldsymbol{\delta}_{\mathbf{2}}=\boldsymbol{\delta}_{\mathbf{1}}+\Delta \mathbf{X}^{(2)}\left(\left(X_{0}, Y_{0}\right)+\boldsymbol{\delta}_{\mathbf{1}}\right)$

In order to find a suitable neighboring shift vector, vectors surrounding $\left(X_{0}, Y_{0}\right)$ are analyzed. For a fixed distance between the second-order vector and the neighboring vector, the angle $\alpha$ is varied (see Fig. 3). The angles $\beta_{1}$ and $\beta_{2}$ depend on $\alpha$ as follows:

$$
\begin{aligned}
\beta_{1}(\alpha)= & -\varphi \\
& +\arctan \left(\frac{r \cdot\left(2 \cdot \sin \alpha-V_{X} \cos \alpha-V_{Y} \sin \alpha\right)}{r \cdot\left(2 \cdot \cos \alpha-U_{X} \cos \alpha-U_{Y} \sin \alpha\right)}\right)
\end{aligned}
$$

$$
\begin{aligned}
\beta_{2}(\alpha)= & \varphi \\
& -\arctan \left(\frac{r \cdot\left(2 \cdot \sin \alpha+V_{X} \cos \alpha+V_{Y} \sin \alpha\right)}{r \cdot\left(2 \cdot \cos \alpha+U_{X} \cos \alpha+U_{Y} \sin \alpha\right)}\right)
\end{aligned}
$$

where $\left(U_{0}, V_{0}\right)$ are the components of the second-order shift vector at $\left(X_{0}, Y_{0}\right)$, and $U_{X}, U_{Y}, V_{X}$, and $V_{Y}$ are the partial derivatives with respect to $X$ and $Y$, respectively. The angle $\varphi$ is the orientation of the second-order shift vector. It is important to note that $\beta_{1}$ and $\beta_{2}$ are not given explicitly in Eq. 2.8 and 2.9. However, it is possible to compute $\beta_{1}(\alpha)$ and $\beta_{2}(\alpha)$ for a set of $\alpha$ (ranging from $\varphi-$ $\pi / 2$ to $\varphi+\pi / 2)$ and find the solution for $\beta_{1}(\alpha)=\beta_{2}(\alpha)$ numerically at fairly low computational cost. Once the angle $\beta_{1}$ is found, the radius $R$ of the circle can be computed

$R=\frac{\left|\Delta \mathbf{X}^{(2)}\left(X_{0}, Y_{0}\right)\right|}{2 \cdot \sin \frac{\xi}{2}}$

where the arc's angle is given by

$\frac{\xi}{2}=\beta_{1}$

The sign of $R$ defines the direction of the curvature: If $R>0$, the streamline follows clockwise rotation, and for $R<0$, the streamline follows counter-clockwise rotation in a right-handed system. Finally, from the radius $R$ and the arc's angle $\xi$, the curvature-correction is applied to the second-order shift vector field in three steps as follows: 
- the second-order vector is shifted toward the streamline with constant curvature using the shift vector $\boldsymbol{\delta}_{\mathrm{cc}}$ :

$$
\boldsymbol{\delta}_{\mathrm{cc}}=R \cdot\left(1-\cos \left[\frac{\xi}{2}\right]\right) \cdot\left(\begin{array}{c}
-\sin \varphi \\
\cos \varphi
\end{array}\right)
$$

- the second-order shift vector is enlarged from the tangent's length to the arc length in order to compensate for the bias error of the absolute value from Eq. 2.1

- the corrected vector field is interpolated to the regular grid of the second-order vector field

It should be emphasized that the estimation of the angle $\beta_{1}$ is based on the gradients of the second-order shift vector field (see Eqs. 2.8 and 2.9). Thus, for reliable curvaturecorrection, the gradients must be estimated accurately first.

For 3D PTV or tomographic PIV, the approach works in the same way: A neighboring shift vector that fulfills Eq. 2.5 allows for the curvature-correction. Additionally, the vectors $\delta_{1}$ and $\delta_{2}$ from Fig. 3 must lie in the same plane in order to estimate the most likely path of motion with constant curvature.

\section{Synthetic example: Lamb-Oseen vortex}

The Lamb-Oseen vortex is a frequently used vortex model in fluid dynamics. The circumferential velocity component $V_{\varphi}$ of this vortex model is given by the following equation:

$V_{\varphi}(r)=\frac{\Gamma}{2 \pi r}\left(1-\exp \left[\frac{-r^{2}}{r_{c}^{2}}\right]\right)$

where $\Gamma$ is the total circulation and $r_{c}$ the vortex core radius. The radial velocity of a Lamb-Oseen vortex is zero; thus, the streamlines have a constant curvature. Therefore, this vortex is well suited to validate the capability of the developed method. The curvature-correction eliminates (in theory) the bias error completely due to constant curved streamlines. Thus, no bias error should be visible after correction. To demonstrate the performance of the developed method, synthetic images were generated, as described in Sect. 3.1, and evaluated using different methods: window-correlation for a single pair of PIV images (Sect. 3.2), ensemble-averaged window-correlation (Sect. 3.3) and single-pixel ensemble-correlation (Sect. 3.4).

\subsection{Image generation}

The analysis based on synthetic images gives full control of all simulation parameters without any limitations regarding their range, and it is possible to detect sensitivities, which indicate what is relevant and what is not (Kähler et al. 2012; Stanislas et al. 2008); 10,000 synthetic image pairs,
$512 \times 512$ px in size, with a stationary Lamb-Oseen vortex in the center, were generated for different total circulations $\Gamma$.

A particle image density of dens $=25 \%$ was applied, meaning that $25 \%$ of the image area was covered by particle images. The digital particle image diameter was set to $D=3 \mathrm{px}$; hence, the number of particles per pixel was $N_{\text {ppp }}=4 \cdot$ dens $/\left(D^{2} \cdot \pi\right)=0.035$ on average. A Gaussian particle image shape was assumed

$I(X, Y)=I_{0} \cdot \exp \left[-8 \cdot\left[\left(\frac{X-X_{0}}{D}\right)^{2}+\left(\frac{Y-Y_{0}}{D}\right)^{2}\right]\right]$

where $\left(X_{0}, Y_{0}\right)$ is the randomly chosen center position, and $D$ is the diameter at $1 / e^{2}$ of the maximum intensity (4 times the standard deviation). The maximum intensity of the particle images was $I_{0}=2^{14}$. From Eq. 3.2, the discrete pixel's gray values were computed from the integral over the pixel's area, corresponding to a sensor fill factor of 1 . Non-uniform pixel response, as outlined in Kähler (2004), was not considered.

The intensity distribution of the first synthetic PIV frame $A$ is given by the following equation:

$A(X, Y)=\sum_{p=1}^{P}\left[\int_{X-0.5}^{X+0.5} \int_{Y-0.5}^{Y+0.5} I_{p}(X, Y) \mathrm{d} x \mathrm{~d} y\right]$

where $P$ and $p$ are the number of particle images and the corresponding control variable, respectively. On top of the particle image intensities, a Gaussian noise with zero mean and a standard deviation of $1 \% \cdot I_{0}$ were added. Finally, the intensity distribution was converted into a 16-bit unsigned integer matrix.

For the second frame, the center positions of the particle images were shifted such that the distances to the vortex center remained constant and the arc lengths were proportional to the circumferential velocity from Eq. 3.1. The image generation was performed using MatLab functions.

\subsection{Window-correlation}

To analyze the effect of curved streamlines on the accuracy of the estimated vector fields, single PIV image pairs of a synthetic vortex are processed. Figure $4 \mathrm{a}$ shows an instantaneous vector field computed with window-correlation using DaVis8.1 (by LaVision GmbH), including multipass evaluation with decreasing window size, iterative window shifting, image deformation and adaptive Gaussian window weighting. The interrogation window size was reduced from $64 \times 64 \mathrm{px}$ down to $16 \times 16 \mathrm{px}$, and two passes were performed for each window size. Furthermore, an initial shift vector field, computed from the product of a 

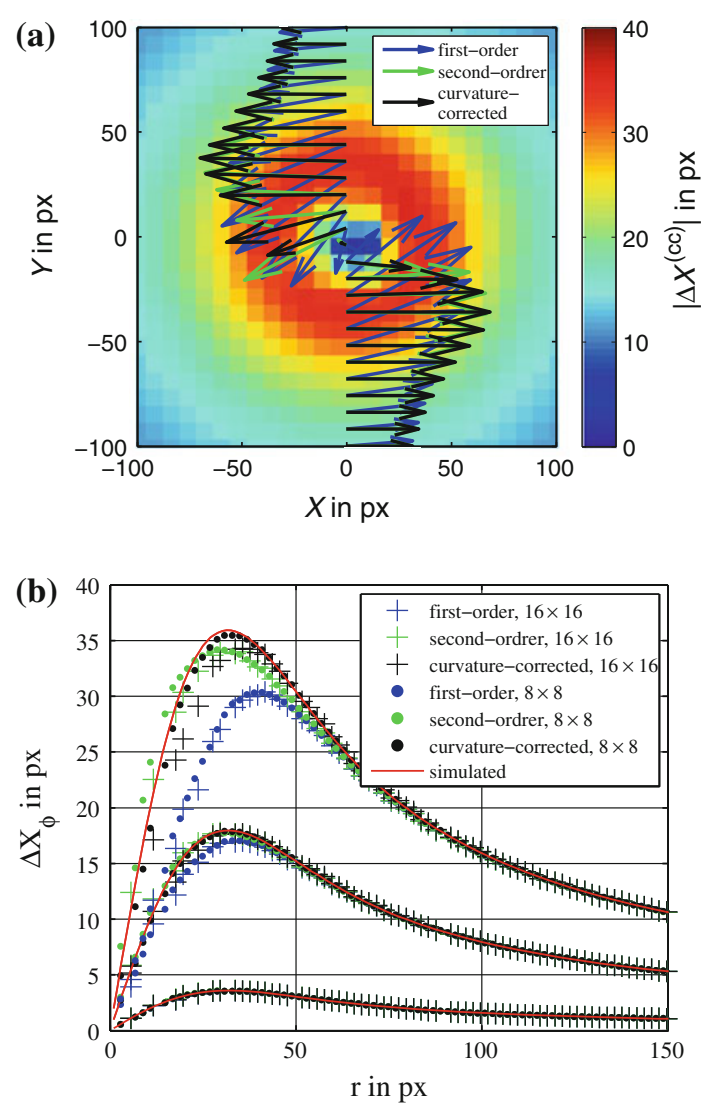

Fig. 4 Instantaneous curvature-corrected shift vector field computed with window-correlation (a) and estimated circumferential velocity component of a simulated Lamb-Oseen vortex (b)

constant and the field of a slowly rotating vortex, was used to overcome the difficulties due to the large shift vector length and the strong gradients. The background color in Fig. 4a represents the absolute value of the curvature-corrected shift vector. Shift vectors with first- and secondorder accuracy as well as curvature-corrected ones are shown in different colors for $X=-4 \mathrm{px}$. The vortex core radius and the total circulation were $r_{c}=20 \mathrm{px}$ and $\Gamma=10^{4} \mathrm{px}^{2}$, respectively. This results in a relatively large maximum particle image shift of $36 \mathrm{px}$. Figure $4 \mathrm{~b}$ shows the circumferential shift vector component $\Delta X_{\Phi}$ for three different circulations $\left(\Gamma=\left[1,000 ; 5,000 ; 10^{4}\right] \mathrm{px}^{2}\right)$ estimated with first- and second-order accuracy and for curvature-corrected vectors using a final interrogation window size of $8 \times 8 \mathrm{px}$ and $16 \times 16 \mathrm{px}$.

It can be concluded from Fig. 4 that for the test case

- for a shift vector length of $<3.6 \mathrm{px}$, the maximum bias error of first-order and second-order evaluation is $0.01 \mathrm{px}$ which is in the order of (or even below) the random error

- for regions with large gradients $(r<30 \mathrm{px})$, small interrogation windows are required for reliable velocity estimations because the bias error due to the spatial averaging is large compared to the bias error caused by the curvature

- the curvature-correction results in significantly decreased bias in the case of large shift vectors for $8 \times 8 \mathrm{px}$ interrogation windows.

\subsection{Averaged window-correlation}

While instantaneous velocity fields are very important for a deeper understanding of the flow physics, statistical values, such as the mean velocity and Reynolds stresses, are required for the validation of numerical flow simulations and for the proof of analytical theories in fluid mechanics. For the estimation of average quantities, the sum-of-correlation approach is more accurate (Wereley and Meinhart 2001). This is because of two reasons: First, the bias error arising from gradients can be reduced as smaller interrogation windows can be applied. Second, the error in estimating the maximum of the correlation is not summed up as in the case of classical cross correlation analysis.

To analyze the effect of curved streamlines on the accuracy of estimated shift vector fields by using sum-ofcorrelation evaluation, 100 PIV image pairs of a synthetic vortex were processed. Figure 5a shows an averaged vector field computed with window-correlation using DaVis8.1 (by LaVision $\mathrm{GmbH}$ ) with the same processing parameters as before. Once again, the vortex core radius and the total circulation were $r_{c}=20 \mathrm{px}$ and $\Gamma=10^{4} \mathrm{px}^{2}$, respectively. Figure $5 \mathrm{~b}$ shows the circumferential shift vector component for three different circulations, estimated with firstand second-order accuracy using $8 \times 8 \mathrm{px}$ interrogation windows for the final pass as well as curvature-corrected results.

Figure $5 \mathrm{a}, \mathrm{b}$ show that the curvature-correction reduces the bias error significantly in the case of large shift vectors. However, for regions with large gradients $(r<30 \mathrm{px})$, a small systematic deviation remains even for the curvaturecorrection. This bias is caused by the final size of the interrogation windows. To reduce this bias error, singlepixel ensemble-correlation analysis is required.

\subsection{Single-pixel ensemble-correlation}

Single-pixel evaluation can be used for a large amount of PIV image pairs and results in significantly improved spatial resolution (Kähler et al. 2012). This is always of importance for the analysis of flow fields with strong gradients. Single-pixel ensemble-correlation was first applied by Westerweel et al (2004) for stationary laminar flows in microfluidics. In the last years, the approach was extended for the analysis of periodic laminar flows (Billy et al. 

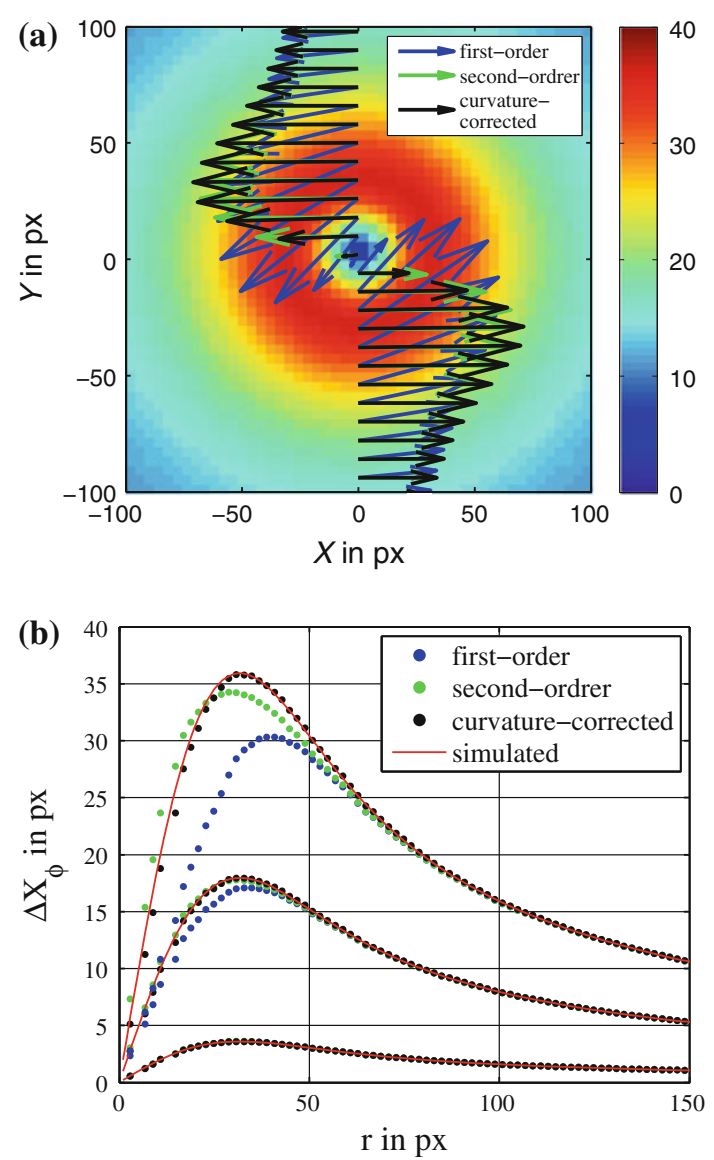

Fig. 5 Averaged curvature-corrected shift vector field computed with sum-of-window-correlation (a) and estimated circumferential velocity component of a simulated Lamb-Oseen vortex (b)

2004), of macroscopic laminar, transitional and turbulent flows (Kähler et al. 2006) and for compressible flows at large Mach numbers (Kähler and Scholz 2006; Bitter et al. 2011). Scholz and Kähler (2006) have extended the highresolution evaluation concept also for stereoscopic PIV recording configurations. Recently, based on the work of (Kähler et al. 2006), the single-pixel evaluation was further expanded to estimate Reynolds stresses in turbulent flows with very high resolution (Scharnowski et al. 2012).

For single-pixel ensemble-correlation, the correlation functions $C(\xi, \psi, X, Y)$ can be computed from a pair of PIV image sets $A(X, Y)$ and $B(X, Y)$ as follows:

$$
\begin{aligned}
& C(\xi, \psi, X, Y) \\
& =\frac{\sum_{n}\left[A_{n}(X, Y)-\bar{A}(X, Y)\right] \cdot\left[B_{n}(X+\xi, Y+\psi)-\bar{B}(X+\xi, Y+\psi)\right]}{\sigma A(X, Y) \cdot \sigma B(X+\xi, Y+\psi)}
\end{aligned}
$$

where the standard deviation is given by:

$\sigma A(X, Y)=\sqrt{\frac{1}{N-1} \sum_{n}\left[A_{n}(X, Y)-\bar{A}(X, Y)\right]^{2}}$
$(X, Y)$ are discrete coordinates of the pixel in question in both images and $(\xi, \psi)$ are the coordinates on the correlation plane. The maximum position of the correlation function $C$ represents the mean shift vector with first-order accuracy. Second-order accuracy can be achieved by moving the first-order vectors to their middle position as demonstrated in Scholz and Kähler (2006). Furthermore, the curvature-correction discussed in Sect. 2.3 can be applied to the second-order vector field (or directly from the first-order field by applying some modifications to Eq. 2.8). In the case of a stationary laminar flow, the curvature-correction estimates the most likely streamline with constant curvature. On the other hand, for turbulent flows, the curvature-correction estimates the mean path with constant curvature which is not identical with the actual streamline of instantaneous vector fields.

In order to analyze the effect of curved streamlines on the accuracy of estimated shift vector fields using singlepixel ensemble-correlation, 10,000 synthetic PIV images were generated and evaluated. Figure 6a shows an averaged vector field computed with single-pixel ensemblecorrelation using Eq. 3.4. As before, the vortex core radius and the total circulation were $r_{c}=20 \mathrm{px}$ and $\Gamma=10^{4} \mathrm{px}^{2}$, respectively. Figure $6 \mathrm{~b}$ shows the circumferential shift vector component for three different circulations estimated with first- and second-order accuracy as well as with curvature-correction. Figure $6 \mathrm{~b}$ shows that the curvature-correction causes the expected bias error reduction for the single-pixel evaluation. Furthermore, even for regions with strong gradients $(r<30 \mathrm{px})$, the third-order accurate data points fit the theoretical curve closely. Thus, the remaining bias of the window-correlation approach could be eliminated completely, as expected from the theory.

Figure 7 shows the difference of the shift vector's absolute value (left) and angle (right) for first-order (top) and second-order (bottom) accuracy with respect to the curvature-corrected shift vector. The simple first-order approximation underestimates the circumferential velocity (Fig. 7a) and causes a strong inward-facing radial component, indicated by the positive sign of the angular bias in Fig. $7 \mathrm{~b}$. The second-order approximation gives the right values for the shift vector's angle (Fig. 7d) but the circumferential component is still biased. Figure $7 \mathrm{c}$ shows that the velocity in the core $(r<20 \mathrm{px})$ is overestimated, while the velocity outside the core is underestimated. The curvature-corrected estimation does not suffer from any bias error for the tested Lamb-Oseen vortex. These findings are in agreement with the theoretical predictions in Sect. 2.

Figure 8 shows the relative bias error of the secondorder shift vector's absolute value with respect to the arc's angle $\xi$. From the figure, it can be concluded that for the tested cases, the bias error is negligible for angles smaller 

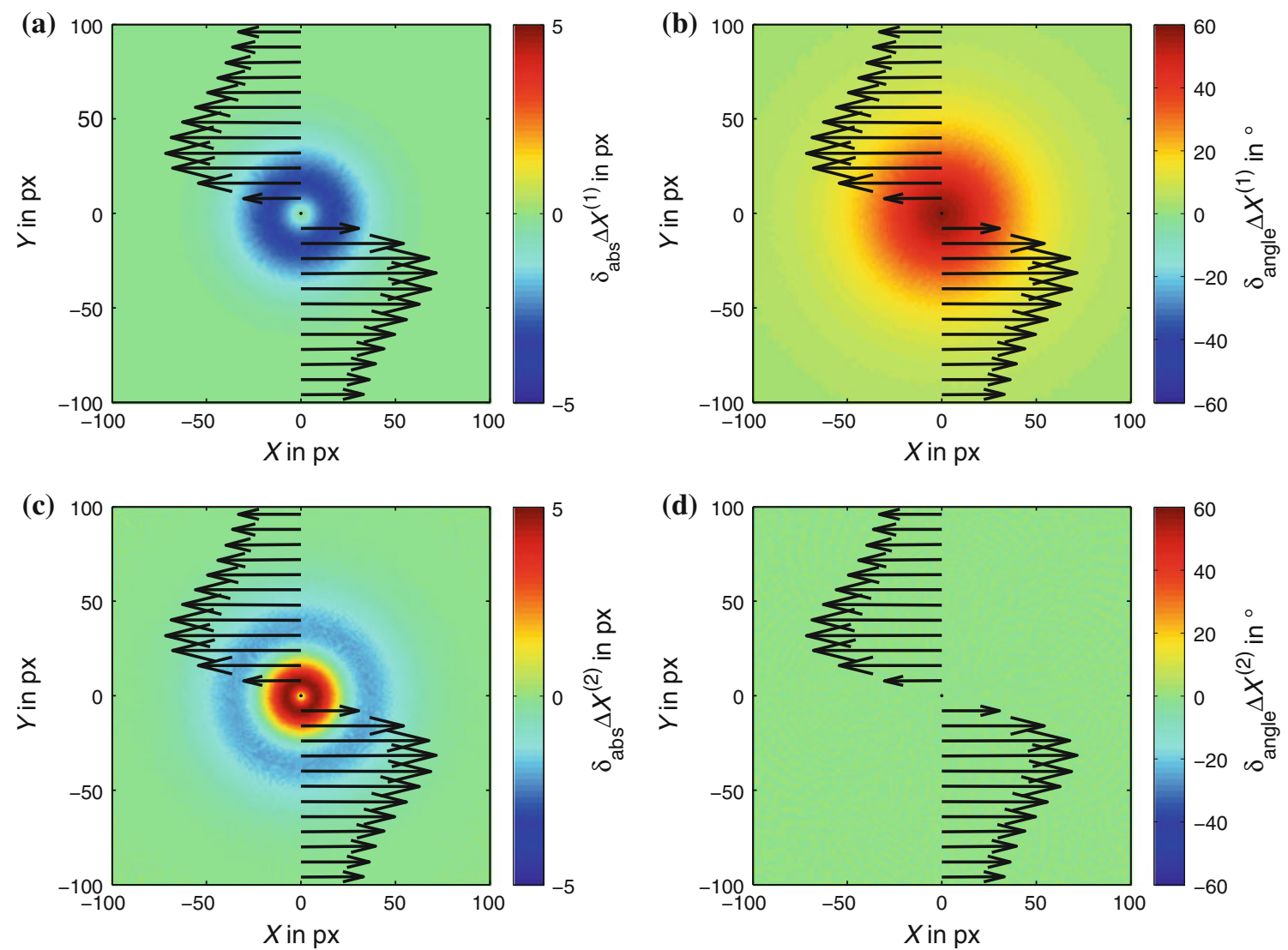

Fig. 6 Bias error of the shift vector's absolute value (left) and angle (right) for first-order (top) and second-order (bottom) accuracy using singlepixel ensemble-correlation

than $\xi<10^{\circ}$. Only for large angles $\xi$, which require strongly curved streamlines, combined with large shift vectors, are the curvature-correction necessary.

\section{Non-constant curvature}

So far, the developed method was applied to synthetic shift vector fields of stationary vortices, where the curvature along the streamlines is constant. This section analyzes vector fields with non-constant curvature. Again, synthetic images are used to have full control of all involved parameters. The test case is a flow along sinusoidal streamlines, as shown in Fig. 9. The shape of a streamline centered at $Y_{0}$ is given as follows:

$Y(X)=a \cdot \sin \left(\frac{X \cdot 2 \pi}{b}\right)+Y_{0}$

where $a$ and $b$ are the amplitude and the period, respectively. The streamline's curvature $1 / R$ changes periodically with respect to $X$
$1 / R=\frac{4 \cdot a \cdot \pi^{2} \sin \left(\frac{2 \cdot \pi \cdot X}{b}\right)}{\left(b^{2}+4 \cdot a^{2} \cdot \pi^{2} \cos ^{2}\left(\frac{2 \cdot \pi \cdot X}{b}\right)\right)^{3 / 2}}$

At the inflection points, the curvature is zero, where it reaches values of up to $4 \cdot a \cdot \pi^{2} / b^{2}$ at the extrema of the sin function in Eq. (4.1). To analyze the effect of this nonconstant curved streamlines on the accuracy of the estimated shift vector field, synthetic image pairs were generated, as discussed in Sect. 3.1. A constant shift vector length $\Delta S$ along the streamlines was simulated. Therefore, for each point $\left(X_{0}, Y_{0}\right)$, the displacement in the $X$ direction $\Delta X$ is computed from the curve's length

$\Delta S=\int_{X_{0}}^{X_{0}+\Delta X} \sqrt{1+\frac{4 \cdot \pi^{2}}{b^{2}} \cdot \cos ^{2}\left(\frac{2 \cdot \pi \cdot X}{b}\right)} d X$

and the displacement in the $Y$ direction from

$\Delta Y=Y\left(X_{0}+\Delta X\right)-Y(\Delta X)$

using the definition in Eq. (4.1). The resulting shift vector field is shown in Fig. 9 for $a=20$ px and $b=128$ px. 

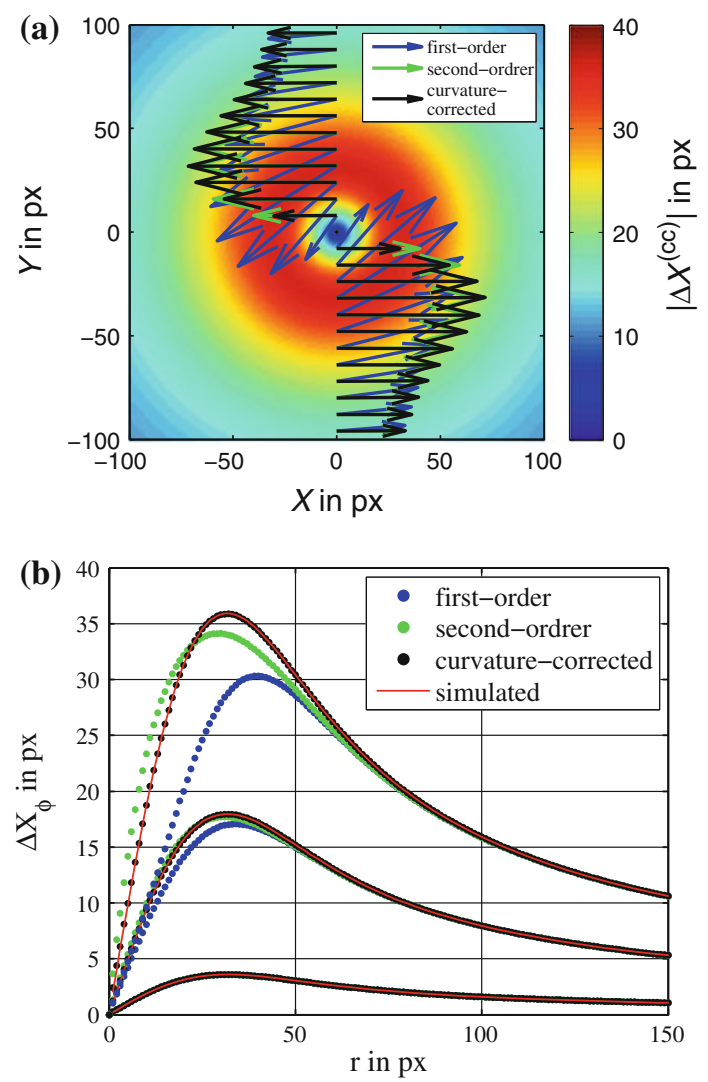

Fig. 7 Averaged curvature-corrected shift vector field computed with single-pixel ensemble-correlation (a) and estimated circumferential velocity component of a simulated Lamb-Oseen vortex (b)

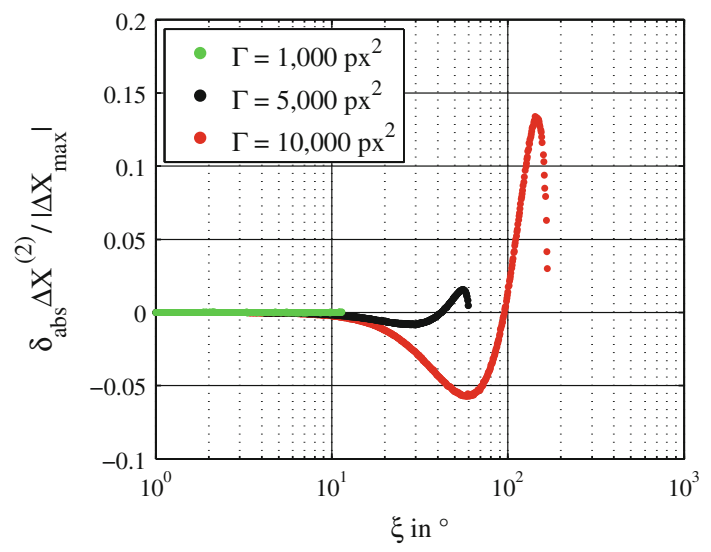

Fig. 8 Bias error of the estimated second-order shift vector's absolute value with respect to the arc's angle

Synthetic PIV image pairs, $256 \times 256$ px in size, with sinusoidal streamlines were generated and evaluated with averaged window-correlation. Figure 10 shows cross section plots of the estimated shift vector length for different simulated $\Delta S$ ranging from 5 to $50 \mathrm{px}$. Each point in Fig. 10 represents the mean displacement (absolute value)

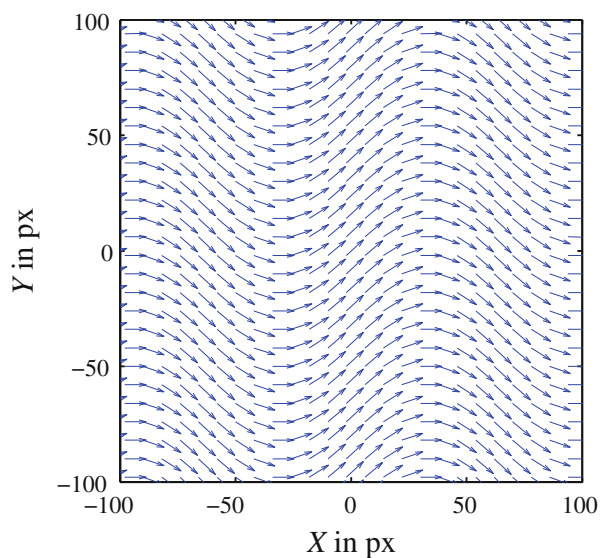

Fig. 9 Synthetic shift vector field with sinusoidal streamlines

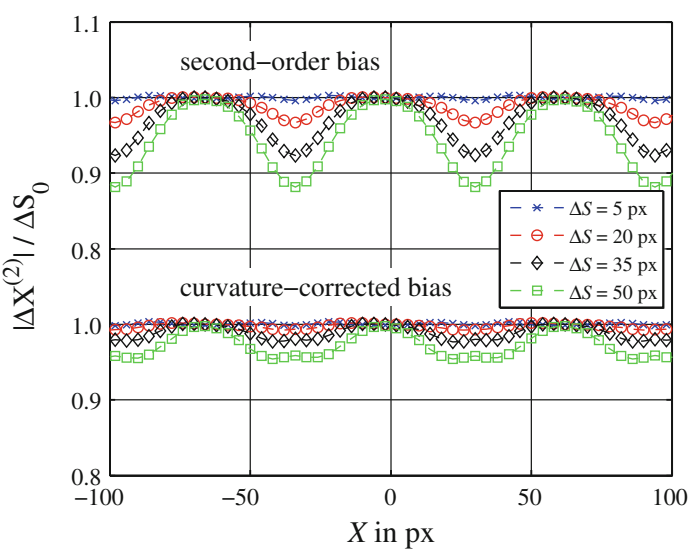

Fig. 10 Bias error of the estimated shift vector's absolute value for a vector field with sinusoidal streamlines computed with second order accuracy and with curvature-correction using average windowcorrelation

averaged over $Y$ computed from 100 image pairs with sumof-window-correlation using a final interrogation window size of $8 \times 8 \mathrm{px}$.

The upper part of Fig. 10 clearly shows a significant bias error: The shift vector length is underestimated in regions with strong curvature. The bias error is largest for large simulated shift vectors and reaches a maximum of $12 \%$ for $\Delta S=50 \mathrm{px}$ at the extrema of the sinusoidal function, which illustrate the importance of this correction for precise measurements. The curvature-correction, shown in the lower part of Fig. 10, results in significantly reduced bias error. The maximum bias error is only $5 \%$. Similar results were achieved by applying window-correlation to single image pairs (resulting in a higher random error) as well as by applying single-pixel ensemble-correlation to 1,000 image pairs.

The mean bias error, averaged over the image area, as a function of the shift vector length is illustrated in Fig. 11; it 


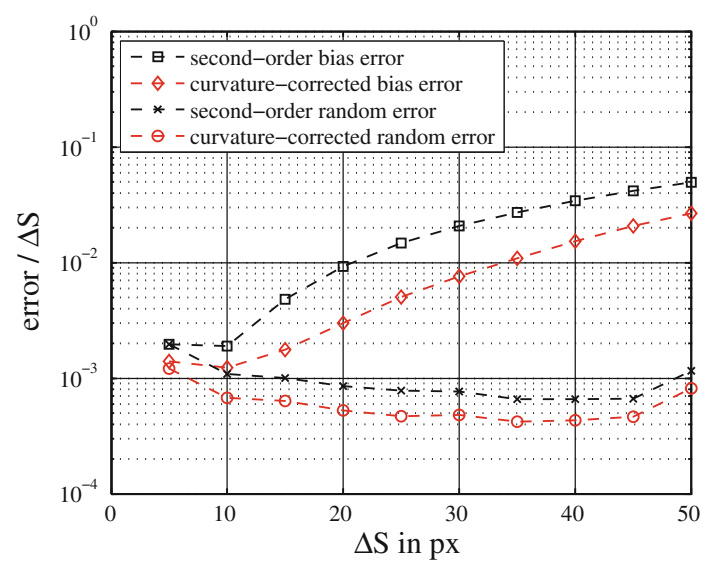

Fig. 11 Mean bias and random error of the second-order accurate shift vectors and of the curvature-corrected ones for a flow field with sinusoidal streamlines

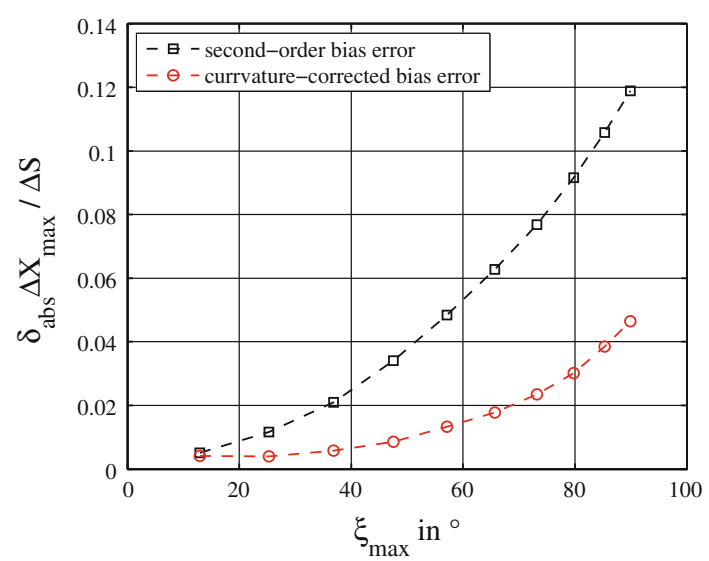

Fig. 12 Maximum bias error of the second-order accurate shift vectors and of the curvature-corrected ones for a flow field with sinusoidal streamlines

can be seen that the curvature-correction results in reduced bias error for all tested shift vector lengths. Additionally, the mean random error is shown in Fig. 11. For the averaged window-correlation of the test case, the mean random error is always lower than the mean bias error. Thus, the shift vector gradients can be reliably detected, and curvature-correction results in improved accuracy.

Figure 12 shows the normalized maximum bias error as a function of the arc's angle $\xi$. The curvature at the extrema of the sinusoidal streamline is relatively large $\left(1 / R_{\min }=\right.$ $\left.4 \cdot a \cdot \pi^{2} / b^{2}=4 \cdot 20 \cdot \pi^{2} / 128^{2} \approx 0.05 \mathrm{px}^{-1}, R_{\min } \approx 20 \mathrm{px}\right)$; thus, the second-order accurate shift vectors are strongly biased. The curvature-correction reduces the maximum bias error significantly. For arc angles $\xi \leq 20^{\circ}$, the maximum bias error of the second-order shift vector is below $1 \%$, which is likely to be smaller than the random error for real PIV images. Thus, curvature-correction becomes important for $\xi>20^{\circ}$.

\section{Flow field example}

In the following, the curvature-correction is applied to a realistic flow field example: test case B of the third PIV challenge (Stanislas et al. 2008). Case B is a synthetic sequence of 120 images with equidistant time intervals of a laminar separation bubble. The image size is $1,440 \times 688$ $\mathrm{px}$, the particle image diameter is around $2 \mathrm{px}$ and the number of particles per pixel is $N_{\mathrm{ppp}}=0.024$.

Images 10 and 11 were evaluated with window-correlation using DaVis8.1 (by LaVision $\mathrm{GmbH}$ ), including multi-pass evaluation with decreasing window size, iterative window shifting, image deformation and adaptive Gaussian window weighting. A final interrogation window size of $16 \times 16 \mathrm{px}$ was chosen. The curvature-correction, as discussed in Sect. 2.3, was applied to the second-order accurate vector field.

Figure 13a shows the estimated curvature-corrected shift vector field. The background color in the figure represents the absolute value of the shift vector, which is characterized by a very large dynamic range. Additionally, characteristic streamlines are drawn in Fig. 13a. Several regions with very strong curvature can be identified from the streamlines. Thus, the curvature-correction approach should help to reduce bias errors.

However, the bias does not only depend on the streamline's curvature but also on the local shift vector length. The arc's angle $\xi$ (see Fig. 2) is an indicator of the necessity of curvature-correction. The spatial distribution of the angle $\xi$ is illustrated in Fig. 13b. It reaches a maximum of $\xi_{\max }=1.75^{\circ}$ at $X=1,140 \mathrm{px}$ and $Y=452$ px. However, according to the findings of Sects. 3 and 4 , values of $\xi<10^{\circ}$ result in negligible bias errors. This is also the case here, as can be seen from the difference of the shift vector length for the second-order accurate vectors and for the curvature-corrected ones, illustrated in Fig. 13c. The estimated maximum bias error is 5 orders (!) of magnitude smaller then the maximum shift vector length. It can be concluded that the developed method of curvature-correction does not improve the accuracy for this particular test case. In order to have a useful correction, the shift vectors must be much larger (factor of 5 or more). This would, on the other hand, increase the amount of loss of paired particle images due to out of plane motion for this 3D flow field. However, for all volumetric PIV and PTV techniques, where out of plane loss of pairs is not possible, this approach can enhance the quality of the results. 
Fig. 13 Test case B2 from the third PIV challenge (Stanislas et al. 2008): Velocity distribution and streamlines (a), covered angle of streamline with respect to the local curvature (b), and difference between the length of secondorder shift vectors and curvature-corrected ones (c)
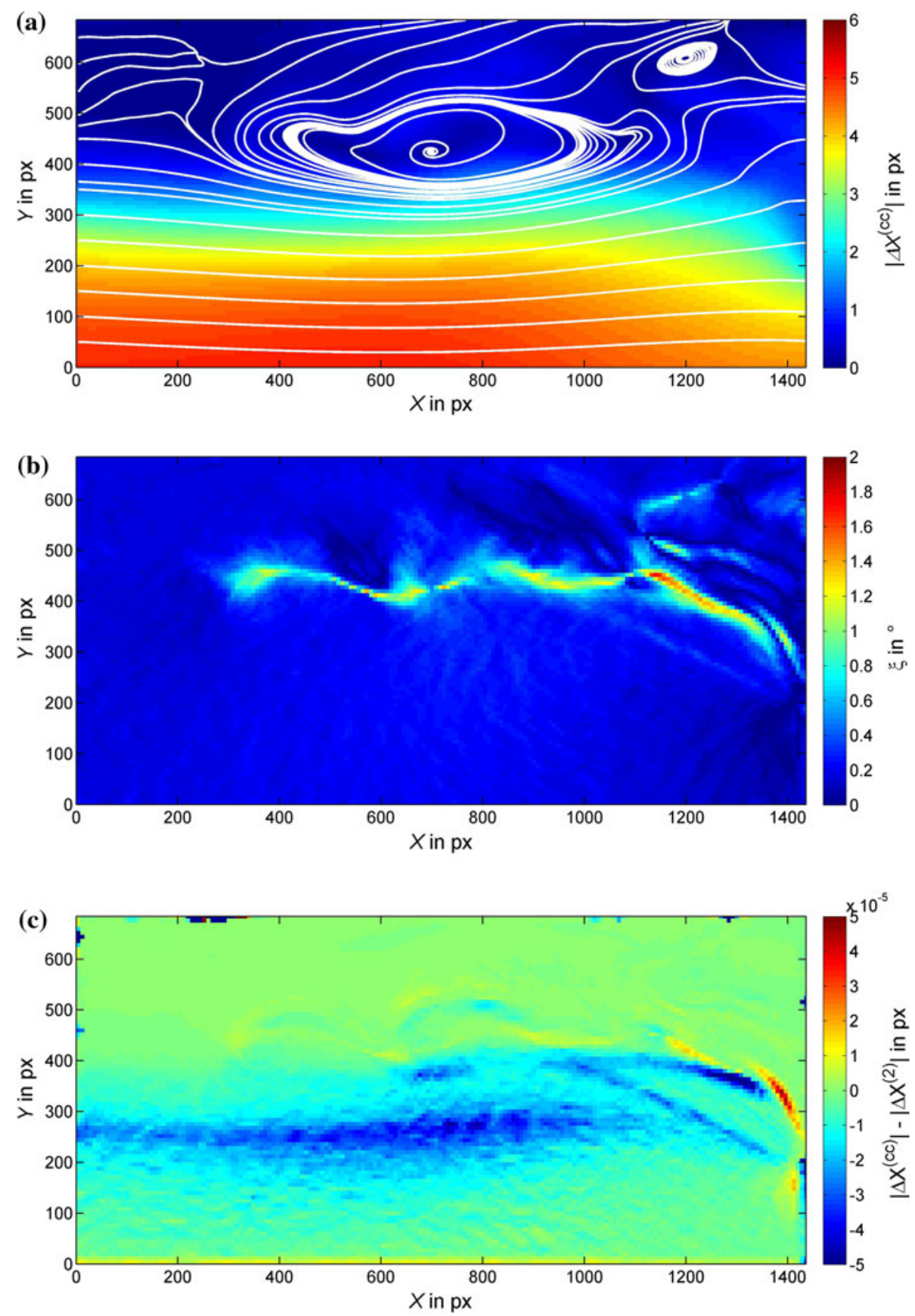

\section{Conclusion}

The presented method allows for the estimation and correction of the bias error due to curved streamlines from PIV data. This result is of great importance for the accurate estimation of velocity fields from PIV data acquired at low optical magnification, as required for high dynamic spatial range. The developed method was validated by analyzing synthetic PIV images of a stationary Lamb-Oseen vortex and of a flow with sinusoidal streamlines. The curvaturecorrected vector fields demonstrated the benefit for standard window-correlation (instantaneous and ensemble-averaged) as well as for single-pixel ensemble-correlation. Due to the curvature-correction, the bias error of the shift vector's absolute value and angle is significantly reduced.

The approach is limited to vector fields with low noise level, since the spatial derivatives of the second-order shift vector must be estimated reliably in order to perform the curvature-correction. Furthermore, the detectable curvature is limited by the averaging area: The interrogation window size must be significantly smaller than the streamline radius for reliable second-order accuracy and curvature-correction. 
The accuracy of a second-order accurate shift vector field that suffers from bias errors due to limited spatial resolution cannot be improved by applying the curvature-correction.

On the other hand, it was shown that the bias error due to curved streamlines is usually negligible for flow fields with moderate shift vector lengths and curvatures $\left(\Delta X<20 \mathrm{px}, \xi<10^{\circ}\right)$. Thus, it can be concluded that second-order accurate PIV evaluation reveals reliable results for most practical applications. However, for highly accurate measurements of the mean velocity field with up to single-pixel resolution, the approach outlined in the paper is recommended to be applied.

Acknowledgments Financial support from the Deutsche Forschungsgemeinschaft (DFG) in the framework of the Sonderforschungsbereich Transregio 40 is gratefully acknowledged by the authors. The authors also would like to thank Rodrigo Segura for technical language revisions.

Open Access This article is distributed under the terms of the Creative Commons Attribution License which permits any use, distribution, and reproduction in any medium, provided the original author(s) and the source are credited.

\section{References}

Adrian RJ (1997) Dynamic ranges of velocity and spatial resolution of particle image velocimetry. Meas Sci Tech 8:1393 doi: 10.1088/0957-0233/8/12/003

Adrian RJ, Westerweel J (2010) Particle image velocimetry. Cambridge University Press, Cambridge

Billy F, David L, Pineau G (2004) Single pixel resolution correlation applied to unsteady flow measurements. Meas Sci Tech 15:1039-1045 doi:10.1088/0957-0233/15/6/002

Bitter M, Scharnowski S, Hain R, Kähler CJ (2011) High-repetitionrate PIV investigations on a generic rocket model in sub- and supersonic flows. Exp Fluids 50:1019-1030 doi:10.1007/s00348010-0988-8

Cierpka C, Lütke B, Kähler CJ (2012) Higher order 4-pulse particle tracking velocimetry. In: 16th international symposium on applications of laser techniques to fluid mechanics, Lisbon, Portugal, July 9-12
Eckstein A, Vlachos PP (2009) Digital particle image velocimetry (DPIV) robust phase correlation. Meas Sci Tech 20:055401. doi: 10.1088/0957-0233/20/5/055401

Hain R, Kähler CJ (2007) Fundamentals of multiframe particle image velocimetry (PIV). Exp Fluids 42:575-587 doi:10.1007/s00348007-0266-6

Kähler CJ (2004) The significance of coherent flow structures for the turbulent mixing in wall-bounded flows. PhD thesis, GeorgAugust-Universität, Göttingen. http://webdoc.sub.gwdg.de/diss/ 2004/kaehler/kaehler.pdf

Kähler CJ, Kompenhans J (2000) Fundamentals of multiple plane stereo PIV. Exp Fluids 29:70-77 doi:10.1007/s003480070009

Kähler CJ, Scholz U (2006) Transonic jet analysis using long-distance micro PIV. In: 12th international symposium on flow visualization, Göttingen, Germany

Kähler CJ, Scholz U, Ortmanns J (2006) Wall-shear-stress and nearwall turbulence measurements up to single pixel resolution by means of long-distance micro-PIV. Exp Fluids 41:327-341 doi: 10.1007/s00348-006-0167-0

Kähler CJ, Scharnowski S, Cierpka C (2012) On the resolution limit of digital particle image velocimetry. Exp Fluids 52:1629-1639. doi:10.1007/s00348-012-1280-x

Lele SK (1991) Compact finite difference schemes with spectral-like resolution. J Comput Phys 103:16-42 doi:10.1016/0021-9991 (92) $90324-R$

Persoons T, O'Donovan TS (2011) High dynamic velocity range particle image velocimetry using multiple pulse separation imaging. Sensors 11:1-18. doi:10.3390/s110100001

Raffel M, Willert CE, Wereley ST, Kompenhans J (2007) Particle image velocimetry: a practical guide. Springer, New York

Scharnowski S, Hain R, Kähler CJ (2012) Reynolds stress estimation up to single-pixel resolution using PIV-measurements. Exp Fluids 52:985-1002 doi:10.1007/s00348-011-1184-1

Scholz U, Kähler CJ (2006) Dynamics of flow structures on heaving and pitching airfoils. In: 13th international symposium on applications of laser techniques to fluid mechanics, Lisbon, Portugal

Sciacchitano A, Scarano F, Wieneke B (2012) Multi-frame pyramid correlation for time-resolved PIV. Exp Fluids 53:1087-1105. doi:10.1007/s00348-012-1345-x

Stanislas M, Okamoto K, Kähler CJ, Westerweel J, Scarano F (2008) Main results of the third international PIV challenge. Exp Fluids 45:27-71 doi:10.1007/s00348-008-0462-z

Wereley ST, Meinhart CD (2001) Second-order accurate particle image velocimetry. Exp Fluids 31(3):258-268 doi:10.1007/ s003480100281

Westerweel J, Geelhoed PF, Lindken R (2004) Single-pixel resolution ensemble correlation for micro-PIV applications. Exp Fluids 37:375-384 doi:10.1007/s00348-004-0826-y 